\title{
OBSERVATION OF THE DYNAMICAL STRUCTURE ARISING FROM SPATIALLY EXTENDED QUANTUM ENTANGLEMENT AND LONG-LIVED QUANTUM COHERENCE IN THE KHCO $\mathrm{KCRYSTAL}_{3}$
}

\author{
François Fillaux $^{1}$, Alain Cousson ${ }^{2}$, David Keen ${ }^{3}$
}

${ }^{1}$ LADIR-CNRS, UMR 7075 Université P. et M. Curie, 2 rue Henry Dunant, 94320 Thiais, France

${ }^{2}$ Laboratoire Léon Brillouin (CEA-CNRS), CEA-Saclay, 91191 Gif-sur-Yvette, cedex, France

${ }^{3}$ ISIS Facility, Rutherford Appleton Laboratory, Chilton, Didcot, OX11 0QX, UK

Physics Department, Oxford University,Clarendon Laboratory, Parks Road, Oxford OX1 3PU, UK

The nonlocal nature of quantum entanglement has remained a subject of great interest since the earliest days of quantum mechanics as it causes many of the paradoxes and lies at the heart of the profound difference between quantum mechanics and classical physics. Quantum entanglement is observed primarily for simple quantum objects (photons or/and atoms) in environments specially designed to minimize quantum decoherence and dissipation.

In the condensed matter, an initially entangled subsystem loses its ability to exhibit quantum interference by getting entangled via interaction with the ambient degrees of freedom. However, the exotic behavior of superconductivity and superfluidity, along with that of laser light, are manifestations of macroscopic quantum effects.

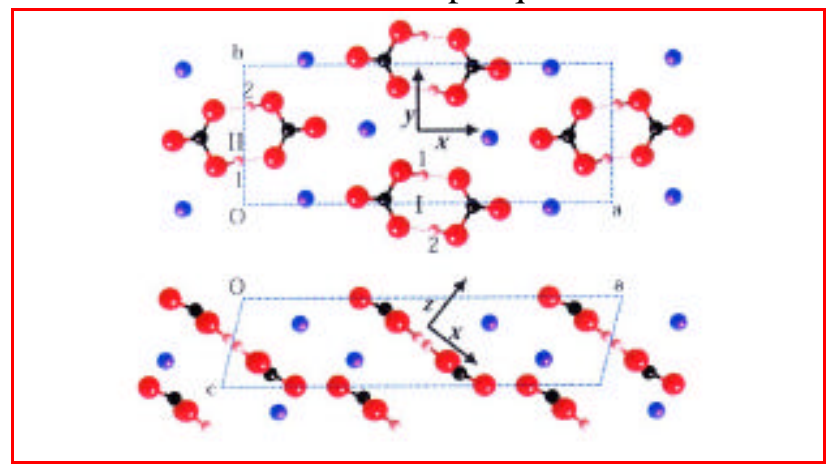

Figure 1. Schematic view of the crystalline structure of $\mathrm{KHCO}_{3}$ at $14 \mathrm{~K}$, measured on $5 \mathrm{C} 2$ at LLB [2]. The dotted lines represent the unit cell. The crystal is monoclinic, space group $P 2_{1} / a\left(\mathrm{C}_{2 \mathrm{~h}}^{3}\right)$, with four $\mathrm{KHCO}_{3}$ entities per unit cell. The dimers at $C i$ sites are parallel to planes at $\approx 42^{\circ}$ with respect to the $(a ; b)$ planes. There is no visible proton disorder. The same conclusion applies to the deuterated derivative.

An ideal situation to observing long-lived quantum entanglement is realized for protons in the potassium hydrogen carbonate crystal $\left(\mathrm{KHCO}_{3}\right.$, Fig. 1) [1]. In this system all protons are equivalent and indistinguishable. The crystal is composed of centrosymmetric dimmers $\left(\mathrm{HCO}_{3}^{-}\right)_{2}$ linked by hydrogen bonds. The local dynamics of protons is represented with symmetric pairs of coupled oscillators. In the degenerate ground state, the Pauli principle imposes antisymmetrization of the vibrational wave function with respect to permutation of the indistinguishable fermions and, consequently, strict separation of the proton dynamics from other atoms [1]. The main decoherence mechanism is forbidden and probing the long-lasting quantum coherence with neutrons gives rise to interference that resemble those commonly observed during double-slit experiments in optic (Fig. 2) [3].

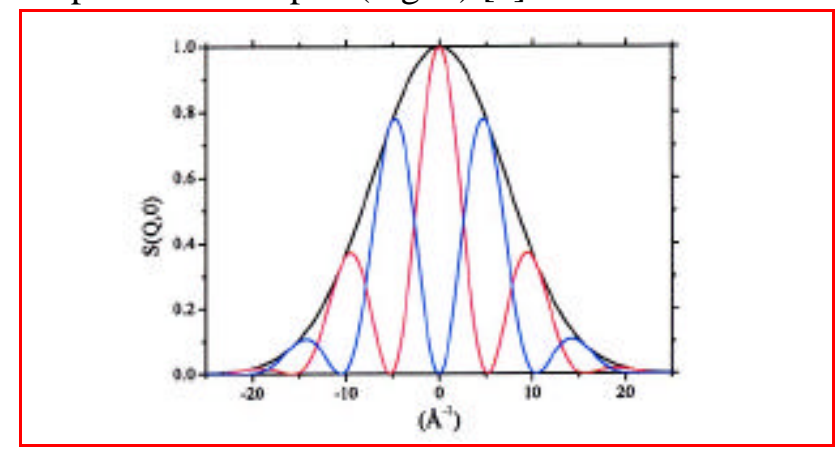

Figure 2. Calculated quantum interference observed with the neutron scattering technique for a pair of protons in $\mathrm{KHCO}_{3}$ at $14 \mathrm{~K}[1,3]$. Black: uncorrelated protons. Red: singlet state for the symmetric mode. Blue: Triplet state for the antisymmetric mode. Similar profiles were observed along the three directions labelled $x, y$ and $z$ in Figure 1

In order to demonstrate the macroscopic character of the quantum entanglement, we have performed neutron diffraction experiments. The diffraction pattern anticipated for the dynamical structure of the sublattice of entangled protons is quite different from the Bragg-peaks of the crystal lattice. The overall shape of the pattern depends on the dimensionality of the quantum correlation. Moreover, the pattern is not determined solely by the spatial distribution of entangled protons. It is also related to the proton dynamics and the symmetry of the phonon states imposes specific constraints to the phase of the scattered neutrons. The intensity is proportional to the total cross section for protons (1/4 80 barns). 


\section{STRUCTURES AND PHASE TRANSITIONS}

With the SXD diffractometer (ISIS, Rutherford Appleton Laboratory, UK) we have measured a rather 2 large volume of the reciprocal space parallel to the $\left(a\right.$ a, $c$ a) plane of $\mathrm{KHCO}_{3}$ and KDCO3 at $15 \mathrm{~K}$ (Fig. 3 and 4, respectively) [2]. Compared to the four-circle diffractometer, the advantage of SXD is twofold. Firstly, with the time-of-flight technique, the whole accessible range of reciprocal space is measured all at once for each neutron pulse. This is convenient for seeking signals in addition to the Bragg intensities. Secondly, with the high flux of epithermal neutrons delivered by the spallation source, one can probe a much larger domain of reciprocal space than at a reactor source.

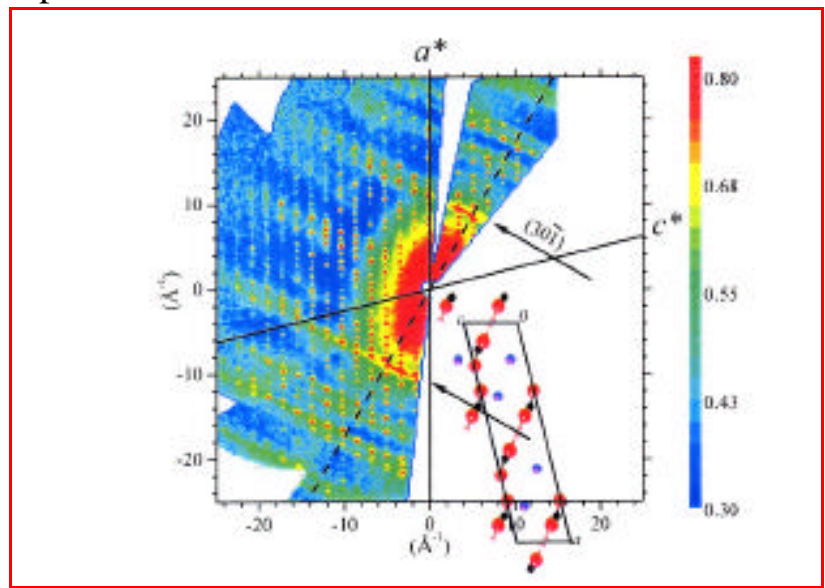

Figure 3. Diffraction pattern in the $(a * ; * a)$ plane of $\mathrm{KHCO}_{3}$ at $15 \mathrm{~K} \mathrm{[2].} \mathrm{The} \mathrm{dash} \mathrm{line} \mathrm{and} \mathrm{the} \mathrm{ridges} \mathrm{of}$ intensity along the $\left(30^{-} 1\right)$ correspond to diffraction parallel and perpendicular to the dimer planes, respectively.

On the other hand, only a limited number of detectors was available on SXD at the time of these measurements. In addition, with the time-offlight technique the data analysis is more complex In the present work, we have gained advantage from both techniques. The structure was determined at the best accuracy with the fourcircles technique and quantum interference were quickly detected in the overview of the reciprocal space obtained with the time-of-flight technique.

For $\mathrm{KHCO}_{3}$, the ridges of intensity along the (301) direction observed at $\pm(10.25 \pm 0.25) \AA^{-1}$

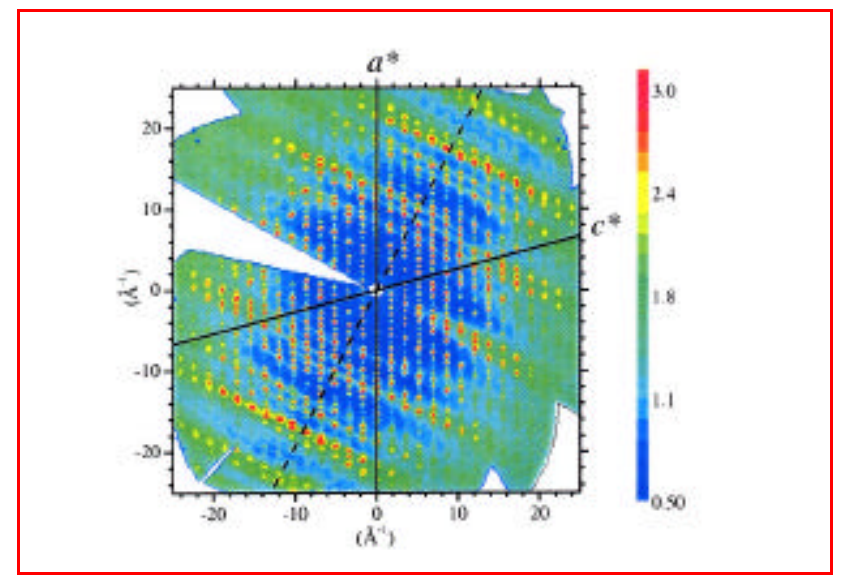

Figure 4. Diffraction pattern in the $\left(a^{*} ; c^{*}\right)$ plane of $\mathrm{KDCO}_{3}$ at $15 \mathrm{~K}$ [2]. The dash line corresponds to diffraction parallelto the dimer planes.

from the center have all the characteristics anticipated for coherent scattering by entangled protons in planes containing the dimer entities: (i) the ridges are not due to coherent scattering by the whole lattice; (ii) they are not observed for the deuterated analogue; (iii) they have rod-like shapes; (iv) orientations and positions are in accord with the structure of the proton sublattice. Additional ripples of diffuse scattering observed at $\approx \pm 17$ and $\pm 22 \AA^{-1}$ for $\mathrm{KHCO}_{3}$ are rather weak and broad. As they survive in the deuterated analogue they are not related to quantum statistics.

In conclusion, $\mathrm{KHCO}_{3}$ can be termed a "quantalcrystal" (or Q'stal). The superposition of fully entangled macroscopic vibrational states with long life-time is a dramatic burst of the quantum paradoxes into the macroscopic world. Compared to superconductivity and superfluidity, the $\mathrm{KHCO}_{3}$ Q'stal shows some remarkable similarities, such as entangled pairs of fermions and largescale longlived quantum coherence in the ground state. Indeed, these similarities are natural consequences of the basic principles of quantum physics. At the same time, the Q'stal is quite different from other macroscopic quantum systems. The entangled pairs are not mobile, they are totally decoupled from the host lattice and the quantum coherence could survive at rather high temperature.

This new state of the matter deserves further investigations.

\section{References}

[1] F. Fillaux, Physica D 113 (1998) 172.

[2] F. Fillaux, A. Cousson, and D. Keen, Phys. Rev. B 67 (2003) 054301.

[3] S. Ikeda and F. Fillaux, Phys. Rev. B 59 (1999) 4134. 